Acta Crystallographica Section D

Biological

Crystallography

ISSN 0907-4449

\section{Ribosome engineering to promote new crystal forms}

\author{
Maria Selmer, ${ }^{a, b_{*}} \neq$ Yong-Gui \\ Gao, $^{b} \ddagger \S$ Albert Weixlbaumer ${ }^{\text {ba }}$ \\ and V. Ramakrishnan ${ }^{b_{*}}$
}

a Department of Cell and Molecular Biology, Uppsala University, Box 596, SE-751 24 Uppsala, Sweden, and ${ }^{\mathbf{b}}$ MRC Laboratory of Molecular Biology, Hills Road, Cambridge $\mathrm{CB} 2 \mathrm{OQH}$, England

₹ These authors contributed equally to the manuscript.

$\S$ Present address: School of Biological Sciences, Nanyang Technological University, 60 Nanyang Drive, Singapore 637551,

Singapore.

- Present address: The Rockefeller University, Box 224, New York, NY 10065, USA.

Correspondence e-mail: maria.selmer@icm.uu.se, ramak@mrc-Imb.cam.ac.uk
Crystallographic studies of the ribosome have provided molecular details of protein synthesis. However, the crystallization of functional complexes of ribosomes with GTPase translation factors proved to be elusive for a decade after the first ribosome structures were determined. Analysis of the packing in different $70 \mathrm{~S}$ ribosome crystal forms revealed that regardless of the species or space group, a contact between ribosomal protein L9 from the large subunit and 16S rRNA in the shoulder of a neighbouring small subunit in the crystal lattice competes with the binding of GTPase elongation factors to this region of $16 \mathrm{~S}$ rRNA. To prevent the formation of this preferred crystal contact, a mutant strain of Thermus thermophilus, HB8-MRCMSAW1, in which the ribosomal protein L9 gene has been truncated was constructed by homologous recombination. Mutant $70 \mathrm{~S}$ ribosomes were used to crystallize and solve the structure of the ribosome with EF-G, GDP and fusidic acid in a previously unobserved crystal form. Subsequent work has shown the usefulness of this strain for crystallization of the ribosome with other GTPase factors.

\section{Introduction}

The ribosome is responsible for the translation of mRNA into protein. Structural understanding of this process was greatly advanced by the determination of the complete structures of the 50S ribosomal subunit from Haloarcula marismortui (Ban et al., 2000) and the 30S ribosomal subunit from Thermus thermophilus (Wimberly et al., 2000). Subsequently, these structures were used to interpret lower resolution structures of the entire $70 \mathrm{~S}$ ribosome in complex with tRNA ligands (Yusupov et al., 2001) as well as with release factors (Petry et al., 2005) and to phase higher resolution structures of the ribosome such as the empty Escherichia coli ribosome (Schuwirth et al., 2005) and the 70S ribosome in complex with tRNA and mRNA (Selmer et al., 2006).

During the translation cycle, GTPase translational factors interact with the ribosome at each of the major stages of initiation (IF2), elongation (EF-Tu and EF-G), termination (RF3) and recycling (EF-G). These factors have all been visualized on the ribosome by cryo-electron microscopy (cryoEM; Connell et al., 2007; Gao et al., 2007; Schuette et al., 2009; Myasnikov et al., 2005; Villa et al., 2009). Despite the increasing resolution of cryo-EM structures, which is currently at about 6-7 А (Schuette et al., 2009; Villa et al., 2009), X-ray crystallography is still the only technique that has been able to directly observe detailed interactions in ribosomal complexes (see, for example, Ogle et al., 2001; Selmer et al., 2006; Hansen et al., 2002). Visualization of the details of these interactions is necessary in order to understand the action of GTPase factors, including the mechanism of GTP hydrolysis and how it is
Received 13 September 2011

Accepted 13 February 2012 
specifically triggered by the ribosome on each factor at the appropriate stage. However, for a decade after the determination of the first structures of ribosomal subunits, the crystallization of ribosomal complexes with translational GTPases proved to be elusive.

Here, we present a crystal-packing analysis of previous $70 \mathrm{~S}$ crystal forms, revealing how preferred crystal contacts involving ribosomal protein L9 compete with the binding of GTPase factors to the ribosome, preventing the crystallization of ribosomal GTPase factor complexes. Furthermore, we describe the rational design of a mutant $T$. thermophilus strain HB8-MRCMSAW1 that cannot form the preferred crystal contact. Finally, we describe how mutant ribosomes from this strain were used to crystallize a $70 \mathrm{~S}$ ribosome complex with EF-G and the antibiotic fusidic acid. This new crystal form allowed structure determination of the EF-G complex (Gao et al., 2009). These mutant ribosomes were also used to crystallize the ribosome with EF-Tu during decoding (Schmeing et al., 2009; Voorhees et al., 2010) and with RF3 during termination (Jin et al., 2011).

\section{Materials and methods}

\subsection{Bacterial strains and plasmids}

T. thermophilus strain HB8 was used as the starting strain for construction of the L9 truncated mutant. A pUC18 clone of the heat-stable kanamycin resistance (HTK) gene (Hoseki et al., 1999) was a kind gift from A. Dahlberg (Brown University). The transformation of $T$. thermophilus was performed as described previously (Koyama et al., 1986).

\subsection{Construction of $T$. thermophilus HB8-MRCMSAW1}

The L9 truncated mutant was prepared using homologous recombination with a pUC19 plasmid containing the HTK gene (Hashimoto et al., 2001; Cameron et al., 2004) flanked by sequences homologous to the region around the L9-encoding rplI gene of the T. thermophilus genome (GenBank accession No. AB103400) according to Fig. 1.

Two fragments of the rplI gene were PCR-amplified using the primers L9f1 (CAAGGTACCGCTTTCCGCCAAGGAGCAGAGGATC; KpnI site shown in bold) and L9b1 (AAAACTGCAGCTAGGCCTGGGCGCGGATCCGG; PstI site shown in bold, stop codon underlined), and L9f2 (AAAACTGCAGCACCATTGACCCCAAGCGCCTGGC; PstI site shown in bold) and L9b2 (GGTAAGCTTCCCCTTGGCCGTGAGCAACCGG; HindIII site shown in bold), and ligated into pUC19 cleaved using KpnI and HindIII. The resulting plasmid was cleaved using PstI and the $H T K$ gene amplified with the primers Htkf (CCACTGCAGGGTACCCGTTGACGGCGGATATGG; PstI site shown in bold) and Htkb (GGTCTGCAGCGTAACCAACATGATTAACAATTATTAGAGG; PstI site shown in bold) was inserted into the Pst site. The resulting plasmid pL9_55_htk was transformed into T. thermophilus HB8 cells (Koyama et al., 1986), which were grown on kanamycin-containing 162 plates to select for recombinants. Incorporation of the truncated L9 gene and the
HTK gene by homologous recombination was confirmed by PCR and sequencing.

\subsection{Preparation of EF-G, $70 S$ and mRNA}

T. thermophilus EF-G was cloned into vector pET42b to create a construct with a C-terminal His tag containing a TEV site and overexpressed in E. coli strain BL21 (DE3). The cells were lysed in lysis buffer $(50 \mathrm{~m} M$ sodium phosphate $\mathrm{pH} 8.0$, $0.3 M \mathrm{NaCl}, 5 \mathrm{~m} M \beta$-mercaptoethanol) using an Emulsiflex (Avestin, Ottawa, Canada) and the cell debris was pelleted by centrifugation for $30 \mathrm{~min}$ at $30000 \mathrm{~g}$. The cell lysate was incubated at $338 \mathrm{~K}$ for $30 \mathrm{~min}$ and denatured E. coli proteins were pelleted by centrifugation. The supernatant was loaded onto an Ni-NTA agarose column (Qiagen) pre-equilibrated in lysis buffer. After washing with lysis buffer containing $20 \mathrm{mM}$ imidazole, EF-G was eluted, dialyzed against TEV buffer $(20 \mathrm{~m} M$ sodium phosphate $\mathrm{pH} 8.0,0.3 \mathrm{M} \mathrm{NaCl}$ ) and treated with TEV protease overnight. After the addition of $20 \mathrm{mM}$ imidazole, the untagged EF-G was passed through an Ni-NTA agarose column (Qiagen). EF-G fractions were pooled and applied onto a HiLoad 26/60 Superdex 200 prep-grade column (Amersham Biosciences) in gel-filtration buffer $(20 \mathrm{mM}$ sodium phosphate $\mathrm{pH}$ 8.0, 0.3 $\mathrm{M} \mathrm{NaCl}$ ). The EF-G peak was

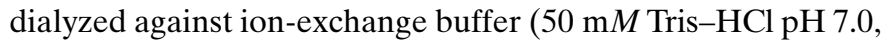
$10 \mathrm{~m} M$ magnesium acetate) and subsequently loaded onto a HiPrep QXL column (GE Healthcare) equilibrated with the same buffer. EF-G was eluted with a linear gradient of 0 $0.7 \mathrm{M} \mathrm{NaCl}$ in ten column volumes. Finally, EF-G was dialyzed against buffer $G$ (5 mM HEPES-KOH pH 7.5, $50 \mathrm{~m} M \mathrm{KCl}$, $10 \mathrm{~m} M$ ammonium chloride, $10 \mathrm{~m} M$ magnesium acetate, $6 \mathrm{mM}$ $\beta$-mercaptoethanol) and concentrated to $24.5 \mathrm{mg} \mathrm{ml}^{-1}$ using an Ultra concentrator (Amicon).

$70 \mathrm{~S}$ ribosomes from $T$. thermophilus HB8-MRCMSAW1 and $E$. coli $\mathrm{RRNA}^{\mathrm{fMet}}$ were prepared using previously described

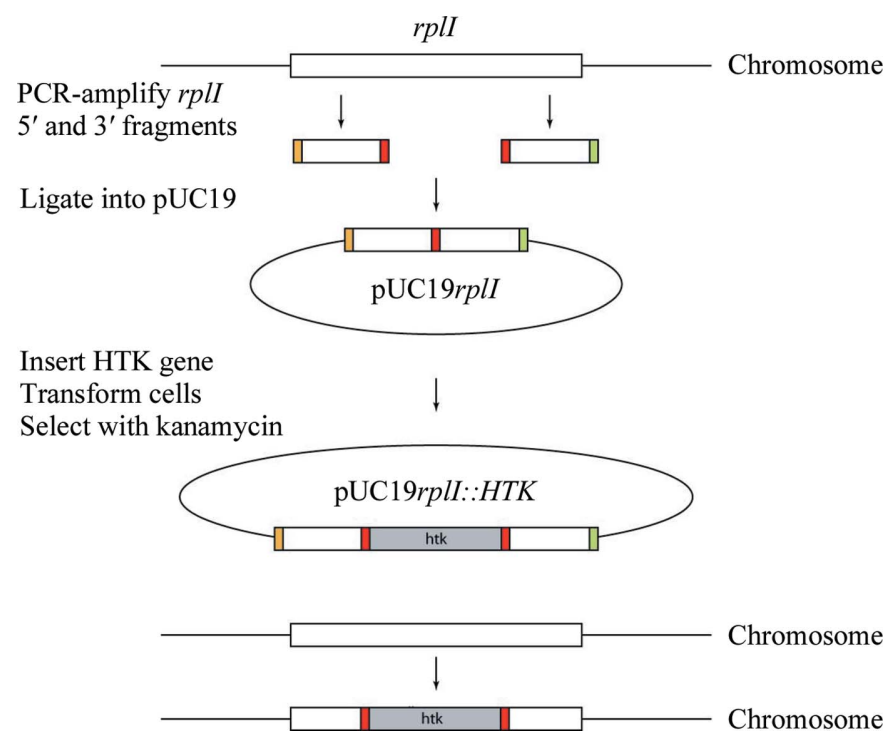

Figure 1

Experimental strategy for the production of T. thermophilus strain HB8MRCMSAW1 using homologous recombination (Hashimoto et al., 2001; Cameron et al., 2004). 
methods (Selmer et al., 2006). The mRNA Z4C was chemically synthesized (Dharmacon) with the sequence 5'-GGCAAGGAGGUAAAAAUGUUCAAAA- ${ }^{\prime}$, with an fMet codon at the $\mathrm{P}$ site (bold) and a Phe codon at the A site (underlined bold).

\subsection{Complex formation, crystallization and structure determination}

$70 \mathrm{~S}$ ribosomes at final concentrations of 4.0 and $8.0 \mu M$ mRNA were incubated in buffer $G$ at $328 \mathrm{~K}$ for 6 min. $16.0 \mu M$ tRNA $^{\text {fMet }}$ was quickly added and the complex was incubated at $328 \mathrm{~K}$ for $6 \mathrm{~min}$. At this point, $500 \mu \mathrm{M}$ fusidic acid, $20 \mu \mathrm{M}$ EF-G and $100 \mu M$ GTP which had been pre-incubated at room temperature for $20 \mathrm{~min}$ were added to the ribosome complex and the resulting mixture was incubated for $20 \mathrm{~min}$ at $328 \mathrm{~K}$ and for $30 \mathrm{~min}$ at room temperature prior to crystallization. 2.3 $\mu M$ Deoxy Big CHAP (DOBC; Hampton Research) was added to the complex, giving a final concentration of $3.3 \mu M$ 70S ribosomes. The complex was subjected to crystallization screening in sitting-drop vapour-diffusion experiments using $200 \mathrm{nl}$ drops (Stock et al., 2005). Initial small crystals grew in sitting drops using Hampton Research Crystal Screen and Crystal Screen 2 (0.1 M MES pH 6.5, 12\% PEG 20K). After optimization to improve the crystal size and quality, $3 \mu \mathrm{l}$ reservoir solution (0.1 $M$ MES pH 6.5-6.6, 8.59.5\% PEG 20K) was mixed with $3 \mu$ complex solution and streak-seeded. Crystals grew in 5-14 d to dimensions of $\sim 20 \times$ $100 \times 500 \mu \mathrm{m}$ (Fig. 2). Data collection, structure determination and refinement have been described elsewhere (Gao et al., 2009).

\section{Results and discussion}

\subsection{Analysis of crystal contacts in the factor-binding site of published 705 ribosomal crystal forms}

In attempts to crystallize the relatively stable complex of the ribosome with EF-G in the presence of the antibiotic

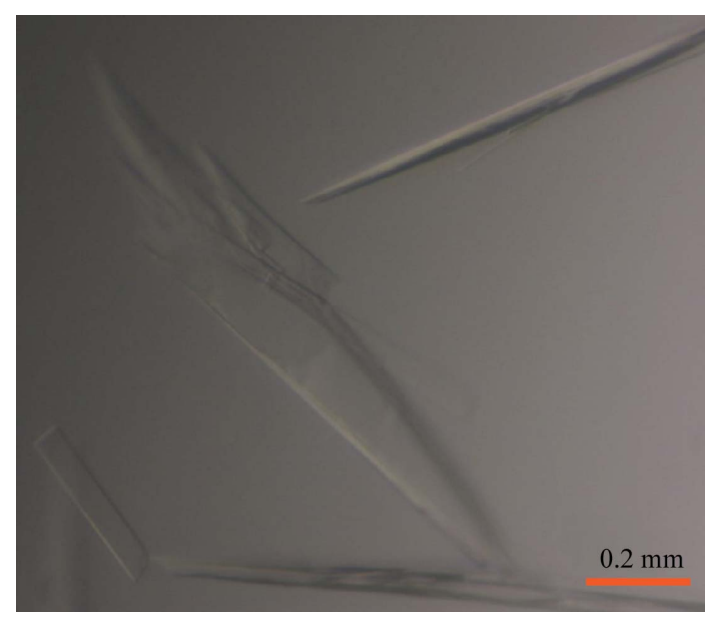

Figure 2

Optimized crystals of the 70S-EF-G complex with GDP and fusidic acid obtained using $\Delta \mathrm{L} 9$ ribosomes. fusidic acid, we obtained diffracting crystals. When the structure was solved, to our disappointment no EF-G was visible. In place of EF-G, L9 of a neighbouring ribosome occupied the factor-binding site of the $30 \mathrm{~S}$ subunit, suggesting that EF-G was competed off the ribosome during crystallization by the L9 crystal contact with the 30 S shoulder (Selmer et al., 2006). This observation led us to check the crystal contacts in other available $70 \mathrm{~S}$ crystal forms.

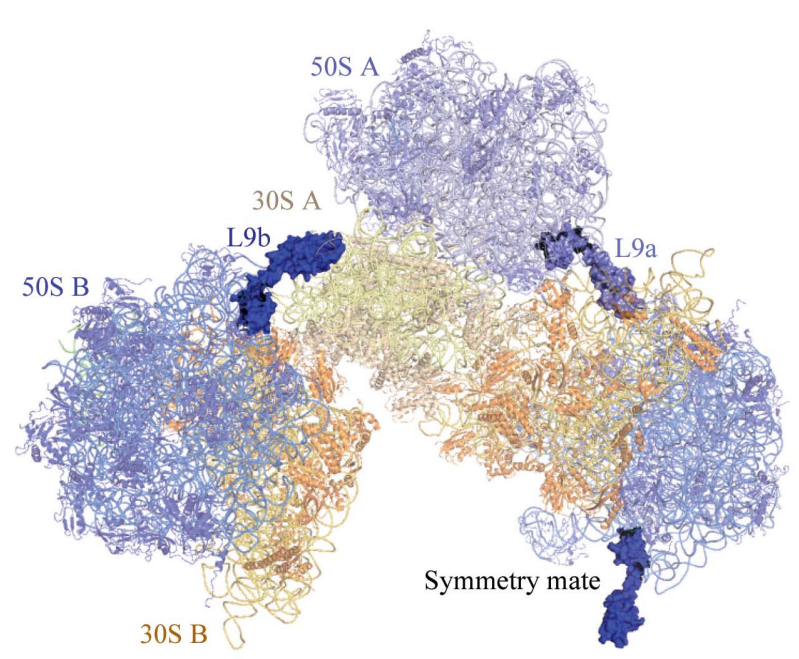

(a)

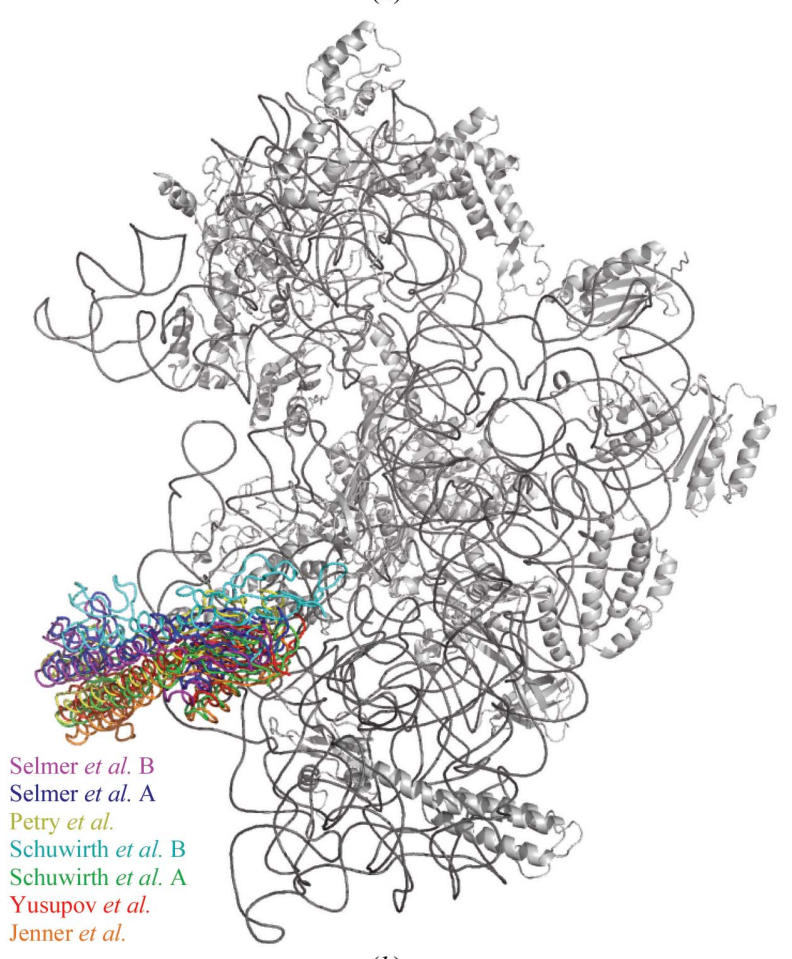

(b)

\section{Figure 3}

(a) Ribosomal protein L9 (dark blue) from one ribosome extends to engage its C-terminal domain in a crystal contact with 16S rRNA of another ribosome, as exemplified by the structure of T. thermophilus $70 \mathrm{~S}$ in complex with mRNA and tRNA (Selmer et al., 2006). 30S proteins and rRNA are shown in yellow. 50S proteins and rRNA are shown in blue. $(b)$ Comparison of the L9 crystal contact with 16S rRNA in different 70 S crystal forms. The structures were superimposed based on the shoulder of 16S rRNA. L9 is shown in different colours in the different structures. 
Table 1

Crystal contacts between ribosomal protein L9 and the 30S shoulder.

\begin{tabular}{llll}
\hline PDB entries and reference & Species and complex & Resolution $(\AA)$ & Hidden surface area $\dagger\left(\AA^{2}\right)$ \\
\hline 2j00, 2j01, 2j02, 2j03 (Selmer et al., 2006) & T. thermophilus 70S, mRNA, 3 tRNAs & 2.8 & L9a-16Sb, 456; L9b-16Sa, 138 \\
1 gix, 1giy (Yusupov et al., 2001) & T. thermophilus 70S, mRNA, 3 tRNAs & 5.5 & n.d. \\
1yl3, 1yl4 (Jenner et al., 2005) & T. thermophilus 70S, mRNA, 2 tRNAs & 5.5 & 462 \\
2avy, 2aw4, 2aw7, 2awb (Schuwirth et al., 2005) & E. coli 70S & 3.5 & L9a-16Sb, 199; L9b-16Sa, 438 \\
2b64, 2b66 (Petry et al., 2005) & T. thermophilus 70S, mRNA, 2 tRNAs, RF1 & 5.9 & 144 \\
\hline
\end{tabular}

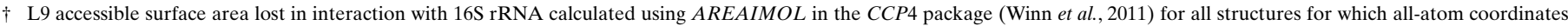
have been deposited in the PDB.

Strikingly, all crystal forms of $70 \mathrm{~S}$ ribosomes in published crystal structures (Petry et al., 2005; Schuwirth et al., 2005; Selmer et al., 2006; Yusupov et al., 2001) had crystal contacts between ribosomal protein L9 in the 50S subunit of one ribosome and the neighbouring $30 \mathrm{~S}$ subunit of another ribosome. L9 consists of two globular domains linked by a long $\alpha$-helix (Hoffman et al., 1994). In all of the different crystal forms, L9 extends from its binding site between 23S rRNA helices $\mathrm{H} 15$ and $\mathrm{H} 76$ below the L1 stalk to engage its C-terminal domain (L9-C) in a crystal contact with the shoulder of the $30 \mathrm{~S}$ subunit of a neighbouring ribosome (Fig. 3a). L9 in a similar conformation extending from the ribosome has also been observed in single-particle cryo-EM studies of $70 \mathrm{~S}$ complexes with EF-G and GTP (Spahn et al., 2001).

Even though the crystal forms belong to different space groups and are from different species, e.g. T. thermophilus (Selmer et al., 2006) or E. coli (Schuwirth et al., 2005), the overall regions of contact between L9 and the neighbouring 30S subunit are similar. The 82-93 and 121-122 loop regions in L9-C contact the 56 and 357-360 regions of 16S rRNA helix 5 and the 368-369 region of 16S rRNA helix 15 using hydrogen bonds and stacking interactions ( $E$. coli rRNA numbering). Despite the similarity, the exact interactions are not conserved: the hidden surface area varies between 140 and $460 \AA^{2}$ and the relative position of the C-terminal domain of L9 differs by up to $20 \AA$. In the three most extensive packing interactions, with more than $400 \AA^{2}$ hidden surface area (Table 1), the position of L9-C differs by about $4 \AA$ (Fig. $3 b$ ).

The L9 contact occurs in the area of the $30 \mathrm{~S}$ subunit where domains II of IF-2 (Myasnikov et al., 2005), EF-G (Connell et al., 2007), EF-Tu (Schuette et al., 2009; Villa et al., 2009) as well as RF3 (Gao et al., 2007) contact helix 5 of 16S rRNA in cryoEM reconstructions of ribosomal complexes with GTPase translation factors. During the translation cycle, the two ribosomal subunits rotate by approximately $6^{\circ}$ with respect to each other in a so-called 'ratcheting' movement. This GTPaseribosome contact occurs in both ratcheted and nonratcheted conformations (Connell et al., 2007; Gao et al., 2007; Schuette et al., 2009; Myasnikov et al., 2005).

Our observations suggested to us that the preferred contact between L9 and the 30 S shoulder could be one of the main causes of the lack of success in crystallizing complexes of 70S ribosomes from several different species with different ribosomal GTPases.

\subsection{Design and construction of a $T$. thermophilus strain with a truncated ribosomal protein $\mathrm{L} 9$ gene}

It has previously been shown that $E$. coli is viable when the chromosomal L9 gene is truncated or deleted and that the peptidyl-transferase activity of $50 \mathrm{~S}$ subunits lacking L9 is indistinguishable from that of the wild type (Lieberman et al., 2000; Herr et al., 2001). The only known function of L9 is in preventing mRNA slippage (reviewed in Atkins \& Björk, 2009). Ribosomes from the bacterium $T$. thermophilus have produced well diffracting crystals of $30 \mathrm{~S}$ subunits (Wimberly et al., 2000) as well as $70 \mathrm{~S}$ ribosomes (Selmer et al., 2006). Therefore, we set out to construct a $T$. thermophilus strain in which ribosomal protein L9 is truncated after the N-terminal domain in order to eliminate its potential crystal contact with a neighbouring $30 \mathrm{~S}$ subunit. The truncation was designed based on the L9 structure within the T. thermophilus $70 \mathrm{~S}$ ribosome (Selmer et al., 2006) and was intended to not perturb the $\mathrm{N}$-terminal 23S RNA-binding domain.

The T. thermophilus HB8-MRCMSAW1 strain was constructed using homologous recombination and has a stop codon after L9 residue 55 followed by a kanamycin-resistance cassette.

\subsection{Crystallization of engineered $T$. thermophilus $70 \mathrm{~S}$ ribosomes in complex with EF-G and analysis of crystal-packing interactions}

Diffraction-quality crystals of the fusidic acid-locked complex of EF-G with $70 \mathrm{~S}$ ribosomes from T. thermophilus strain HB8-MRCMSAW1, mRNA and P-site tRNA were grown in MES buffer $\mathrm{pH}$ 6.5-6.6 using PEG 20K as a precipitant (Fig. 2). There is no electron density for the N-terminal domain of L9 in these structures, indicating that the truncated version of the protein has not been incorporated into the ribosomes of the mutant strain (Gao et al., 2009).

The DOBC detergent, which was added to the complex solution prior to crystallization, was critical for crystal growth in the above condition. Interestingly, the same detergent was also used in the crystallization of wild-type T. thermophilus $70 \mathrm{~S}$ ribosomes with mRNA and tRNA (Selmer et al., 2006), in which case detergent addition improved crystal morphology and diffraction. Using similar but not identical conditions, ribosomes lacking L9 could also be crystallized in complex with EF-Tu (Schmeing et al., 2009; Voorhees et al., 2010) and with RF3 (Jin et al., 2011). 
In the crystal structures of EF-G (Gao et al., 2009) and EF-Tu (Schmeing et al., 2009) bound to the engineered 70S ribosome, as observed in cryo-EM reconstructions, domain II of the elongation factors interacts with helix 5 and helix 15 of the 16S rRNA shoulder (Fig. 4). The exact interactions are not conserved between the fusidic acid-stalled EF-G complex and the kirromycin-stalled EF-Tu complex. The hidden surface area in the contact varies between 481 and $536 \AA^{2}$ and the position of domain II of the two factors differs by $3-5 \AA$
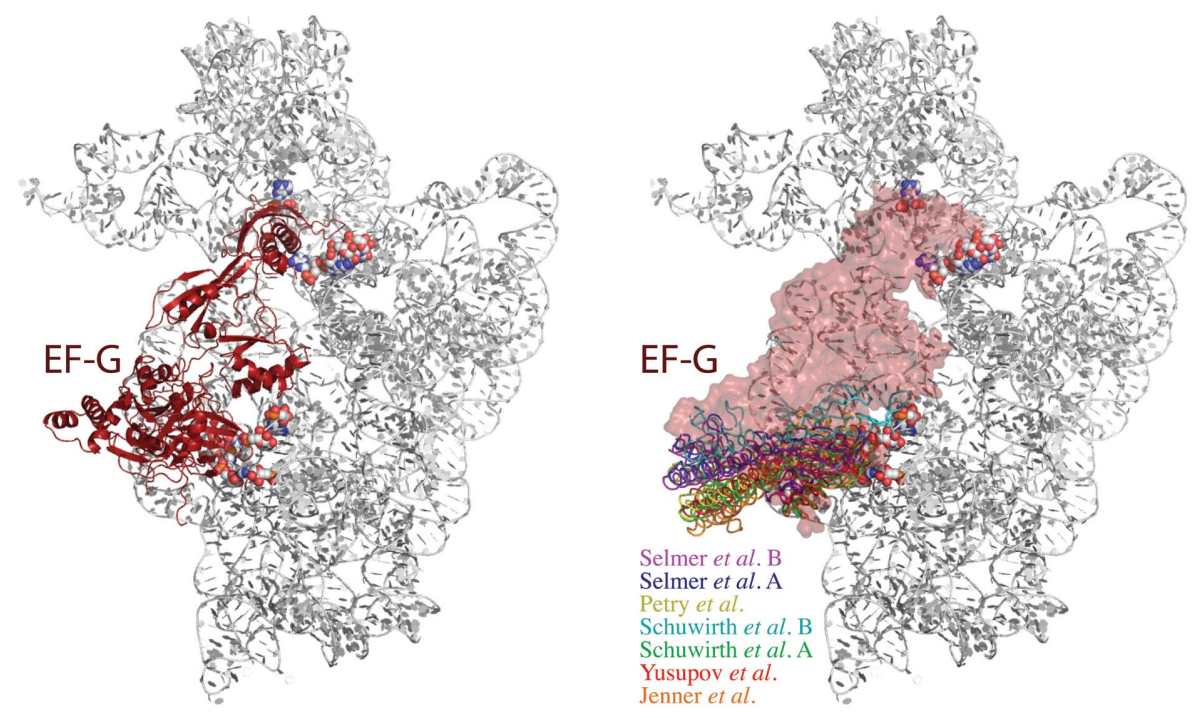

(a)
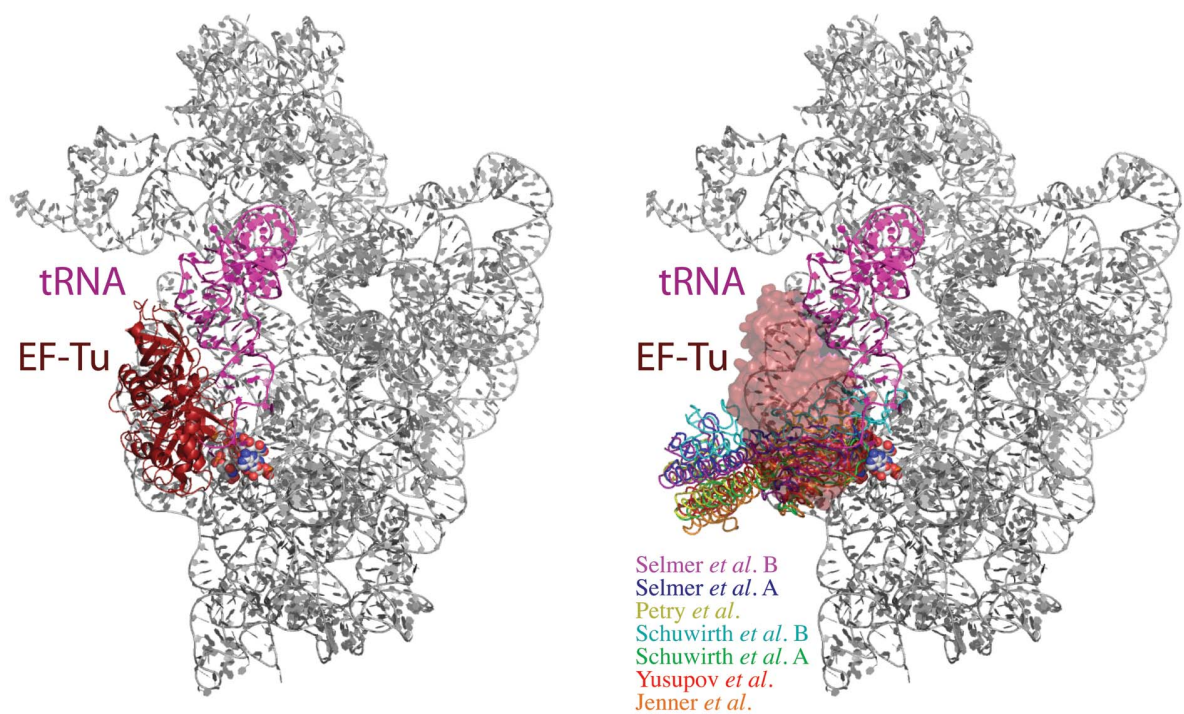

(b)

\section{Figure 4}

Comparison of elongation-factor interactions with the 16S rRNA shoulder and L9 crystal-packing interactions with the same region. (a) Fusidic acid-locked structure of EF-G in complex with the $70 \mathrm{~S}$ ribosome (Gao et al., 2009). In the left panel, EF-G is shown as a brown ribbon and the 30S ribosome is shown in grey. In the right panel, the EF-G surface is shown in transparent brown, the $30 \mathrm{~S}$ ribosome in grey and the L9 crystal contacts in the same colours as in Fig. 3(b). (b) Kirromycinlocked structure of EF-Tu and tRNA with the 70S ribosome (Schmeing et al., 2009). In the left panel, EF-Tu is shown as a brown ribbon, tRNA is shown in magenta and the $30 \mathrm{~S}$ ribosome in grey. In the right panel, the EF-Tu surface is shown in transparent brown, the $30 \mathrm{~S}$ ribosome in grey and the L9 crystal contacts in the same colours as in Fig. 3(b). relative to 16S rRNA. For EF-Tu, this interaction has been implicated in GTPase activation in response to correct decoding (Schmeing et al., 2009) and the same may be true for EF-G (Gao et al., 2009).

This work shows that there are situations in which fortuitous crystal contacts in the ribosome can be so strong that they can displace normal binding of factors and lead to crystallization without the factors present. We had previously been unable to obtain alternative crystal forms that included the factor, presumably because crystallization of the factorless forms was favoured by the L9 contact and drove the equilibrium in this direction. The fact that a deletion mutant unable to form the L9 contact could be used to crystallize three different GTPase ribosome complexes shows that engineering ribosomes to remove favourable contacts can allow the crystallization of completely new forms. It is possible that this strategy may be of use in the crystallization of other macromolecular complexes.

The authors wish to express their gratitude to Steven Gregory, Brown University for helpful advice on $T$. thermophilus genetics, to Christine Dunham for helpful discussions and to Ann Kelley for ribosome purification. MS was supported by the WennerGren foundations, the Swedish Research council and the Swedish foundation for Strategic Research. VR was supported by the UK Medical Research Council, a Wellcome Trust program grant, and awards from the Agouron Institute and the LouisJeantet Foundation.

\section{References}

Atkins, J. F. \& Björk, G. R. (2009). Microbiol. Mol. Biol. Rev. 73, 178-210.

Ban, N., Nissen, P., Hansen, J., Moore, P. B. \& Steitz, T. A. (2000). Science, 289, 905920.

Cameron, D. M., Gregory, S. T., Thompson, J., Suh, M. J., Limbach, P. A. \& Dahlberg, A. E. (2004). J. Bacteriol. 186, 5819-5825.

Connell, S. R., Takemoto, C., Wilson, D. N., Wang, H., Murayama, K., Terada, T., Shirouzu, M., Rost, M., Schüler, M., Giesebrecht, J., Dabrowski, M., Mielke, T., Fucini, P., Yokoyama, S. \& Spahn, C. M. (2007). Mol. Cell, 25, 751-764.

Gao, H., Zhou, Z., Rawat, U., Huang, C., Bouakaz, L., Wang, C., Cheng, Z., Liu, Y., Zavialov, A., Gursky, R., Sanyal, S., 
Ehrenberg, M., Frank, J. \& Song, H. (2007). Cell, 129, 929-941.

Gao, Y.-G., Selmer, M., Dunham, C. M., Weixlbaumer, A., Kelley, A. C. \& Ramakrishnan, V. (2009). Science, 326, 694-699.

Hansen, J. L., Schmeing, T. M., Moore, P. B. \& Steitz, T. A. (2002). Proc. Natl Acad. Sci. USA, 99, 11670-11675.

Hashimoto, Y., Yano, T., Kuramitsu, S. \& Kagamiyama, H. (2001). FEBS Lett. 506, 231-234.

Herr, A. J., Nelson, C. C., Wills, N. M., Gesteland, R. F. \& Atkins, J. F. (2001). J. Mol. Biol. 309, 1029-1048.

Hoffman, D. W., Davies, C., Gerchman, S. E., Kycia, J. H., Porter, S. J., White, S. W. \& Ramakrishnan, V. (1994). EMBO J. 13, 205-212.

Hoseki, J., Yano, T., Koyama, Y., Kuramitsu, S. \& Kagamiyama, H. (1999). J. Biochem. 126, 951-956.

Jenner, L., Romby, P., Rees, B., Schulze-Briese, C., Springer, M., Ehresmann, C., Ehresmann, B., Moras, D., Yusupova, G. \& Yusupov, M. (2005). Science, 308, 120-123.

Jin, H., Kelley, A. C. \& Ramakrishnan, V. (2011). Proc. Natl Acad. Sci. USA, 108, 15798-15803.

Koyama, Y., Hoshino, T., Tomizuka, N. \& Furukawa, K. (1986). J. Bacteriol. 166, 338-340.

Lieberman, K. R., Firpo, M. A., Herr, A. J., Nguyenle, T., Atkins, J. F., Gesteland, R. F. \& Noller, H. F. (2000). J. Mol. Biol. 297, 11291143.

Myasnikov, A. G., Marzi, S., Simonetti, A., Giuliodori, A. M., Gualerzi, C. O., Yusupova, G., Yusupov, M. \& Klaholz, B. P. (2005). Nature Struct. Mol. Biol. 12, 1145-1149.

Ogle, J. M., Brodersen, D. E., Clemons, W. M. Jr, Tarry, M. J., Carter, A. P. \& Ramakrishnan, V. (2001). Science, 292, 897-902.

Petry, S., Brodersen, D. E., Murphy, F. V., Dunham, C. M., Selmer, M., Tarry, M. J., Kelley, A. C. \& Ramakrishnan, V. (2005). Cell, 123, 1255-1266.
Schmeing, T. M., Voorhees, R. M., Kelley, A. C., Gao, Y.-G., Murphy, F. V. IV, Weir, J. R. \& Ramakrishnan, V. (2009). Science, 326, 688-694.

Schuette, J. C., Murphy, F. V., Kelley, A. C., Weir, J. R., Giesebrecht, J., Connell, S. R., Loerke, J., Mielke, T., Zhang, W., Penczek, P. A., Ramakrishnan, V. \& Spahn, C. M. (2009). EMBO J. 28, 755765.

Schuwirth, B. S., Borovinskaya, M. A., Hau, C. W., Zhang, W., VilaSanjurjo, A., Holton, J. M. \& Cate, J. H. (2005). Science, 310, 827-834.

Selmer, M., Dunham, C. M., Murphy, F. V., Weixlbaumer, A., Petry, S., Kelley, A. C., Weir, J. R. \& Ramakrishnan, V. (2006). Science, 313, 1935-1942.

Spahn, C. M., Blaha, G., Agrawal, R. K., Penczek, P., Grassucci, R. A., Trieber, C. A., Connell, S. R., Taylor, D. E., Nierhaus, K. H. \& Frank, J. (2001). Mol. Cell, 7, 1037-1045.

Stock, D., Perisic, O. \& Löwe, J. (2005). Prog. Biophys. Mol. Biol. 88, 311-327.

Villa, E., Sengupta, J., Trabuco, L. G., LeBarron, J., Baxter, W. T., Shaikh, T. R., Grassucci, R. A., Nissen, P., Ehrenberg, M., Schulten, K. \& Frank, J. (2009). Proc. Natl Acad. Sci. USA, 106, $1063-$ 1068.

Voorhees, R. M., Schmeing, T. M., Kelley, A. C. \& Ramakrishnan, V. (2010). Science, 330, 835-838.

Wimberly, B. T., Brodersen, D. E., Clemons, W. M., Morgan-Warren, R. J., Carter, A. P., Vonrhein, C., Hartsch, T. \& Ramakrishnan, V. (2000). Nature (London), 407, 327-339.

Winn, M. D. et al. (2011). Acta Cryst. D67, 235-242.

Yusupov, M. M., Yusupova, G. Z., Baucom, A., Lieberman, K., Earnest, T. N., Cate, J. H. \& Noller, H. F. (2001). Science, 292, 883-896. 